\title{
Circulating MicroRNA-30d is Associated with Response to Cardiac Resynchronization Therapy in Heart Failure and Regulates Cardiomyocyte Apoptosis: A Translational Pilot Study
}

\author{
Yonathan F. Melman, MD, PhD ${ }^{\# 1}$, Ravi Shah, MD ${ }^{\# 1,2}$, Kirsty Danielson, PhD $^{\# 1}$, Junjie Xiao, \\ MD, PhD\#3, Bridget Simonson, $\mathrm{PhD}^{1}$, Andreas Barth, MD ${ }^{4}$, Khalid Chakir, PhD $^{4}$, Gregory D. \\ Lewis, MD², Zachary Lavender, BA ${ }^{2}$, Quynh A. Truong, MD², Andre Kleber, MD ${ }^{1}$, Ranendra \\ Das, $\mathrm{PhD}^{5}$, Anthony Rosenzweig, $\mathbf{M D}^{1}$, Yaoyu Wang, $\mathrm{PhD}^{6}$, David Kass, $\mathbf{M D}^{4}$, Jagmeet $\mathbf{P}$. \\ Singh, MD, $\mathrm{PhD}^{2}$, and Saumya Das, MD, $\mathrm{PhD}^{1}$ \\ ${ }^{1}$ Cardiovascular Division, Beth Israel Deaconess Medical Center, Boston, MA \\ ${ }^{2}$ Cardiology Division, Massachusetts General Hospital, Boston, MA \\ ${ }^{3}$ Experimental Center of Life Sciences and Regeneration Lab, School of Life Science, Shanghai \\ University, Shanghai, China \\ ${ }^{4}$ The Johns Hopkins University Medical Institutions, Baltimore, MD \\ 5 Johns Hopkins University, Baltimore MD \\ ${ }^{6}$ Dana Farber Cancer Institute, Center for Computational Biology, Boston MA \\ \# These authors contributed equally to this work.
}

\begin{abstract}
Background-Biomarkers that predict response to cardiac resynchronization therapy (CRT) in heart failure patients with dyssynchrony $\left(\mathrm{HF}_{\mathrm{DYS}}\right)$ would be clinically important. Circulating extracellular microRNAs (miRNAs) have emerged as novel biomarkers that may also play important functional roles, but their relevance as markers for CRT response has not been examined.
\end{abstract}

\begin{abstract}
Methods and Results—Comprehensive miRNA PCR arrays were used to assess baseline levels of 766 plasma miRNA in patients undergoing clinically indicated CRT in an initial discovery set $(\mathrm{n}=12)$ with and without subsequent echocardiographic improvement at 6 months after CRT. Validation of candidate miRNAs in 61 additional patients confirmed that baseline plasma miR-30d was associated with CRT response (defined as increase in LVEF $\geq 10 \%$ ). MiR-30d was enriched in coronary sinus (CS) blood and increased in late-contracting myocardium in a canine model of $\mathrm{HF}_{\mathrm{DYS}}$, indicating cardiac origin with maximal expression in areas of high mechanical stress. We examined the functional effects of miR-30d in cultured cardiomyocytes (CMs), and determined that miR-30d is expressed in CMs and released in vesicles in response to mechanical stress. Overexpression of miR-30d in cultured CMs led to CM growth and protected
\end{abstract}

Correspondence: Saumya Das, MD, PhD, Cardiovascular Division, Beth Israel Deaconess Medical Center, 330 Brookline Avenue, CLS 907, Boston, MA 02215, Phone: 617-735-4241, Fax: 617-667-7620, sdas@ bidmc.harvard.edu.

Disclosures: None. 
against apoptosis by targeting the mitogen-associated kinase 4 (MAP4K4), a downstream effector of tumor necrosis factor (TNF). In $\mathrm{HF}_{\mathrm{DYs}}$ patients, miR-30d plasma levels inversely correlated with high sensitivity Troponin $\mathrm{T}$, a marker of myocardial necrosis.

Conclusions-Baseline plasma miR-30d level is associated with response to CRT in HF this translational pilot study. MiR-30d increase in CMs correlates with areas of increased wall stress in $\mathrm{HF}_{\mathrm{DYS}}$, and is protective against deleterious TNF signaling.

\section{Keywords}

heart failure; remodeling; dyssynchrony; extracellular RNA

\section{INTRODUCTION}

Ventricular "dyssynchrony" due to delayed activation of the left ventricular (LV) lateral wall is present in nearly $50 \%$ of patients with symptomatic advanced heart failure (HF) ${ }^{1}$ and reduces effective LV function ${ }^{2}$. Cardiac resynchronization therapy (CRT) treats electrical dyssynchrony and mitigates the adverse molecular processes as well as the progressive decline in $\mathrm{LV}$ function and poor prognosis in patients with $\mathrm{HF}^{3,4}$. Unfortunately, even in patients who meet consensus criteria for CRT, 30\% or more do not derive hemodynamic or clinical benefit ${ }^{5}$, and efforts to define clinical, image-based, plasma or electrocardiographic biomarkers to predict responsive patients have failed to lower this percentage ${ }^{67},{ }^{8}$.

Therefore, discovery of novel markers to predict response to CRT more effectively is an urgent clinical need, and may provide additional mechanistic insights into dyssynchrony and LV remodeling.

MicroRNAs (miRNAs) regulate gene networks, playing an important role in cardiovascular diseases including fibrosis, atherosclerosis and arrhythmias ${ }^{9}$. MiRNAs have been implicated in the pathogenesis of HF, and show potential as therapeutic targets in models of $\mathrm{HF}^{10}{ }^{11}$. More recently, miRNAs have been found to be stably present in biofluids, often packaged in extracellular vesicles ${ }^{12}$. Consequently, miRNAs have emerged as biomarkers of cardiovascular disease ${ }^{13}$. Interestingly, recent studies have suggested a functional role for some extracellular miRNAs in disease pathogenesis, however the origin and role of such miRNAs in cardiac remodeling has not been delineated.

To identify circulating miRNAs that are associated with response to CRT, we assessed preimplant plasma miRNA profiles in patients with advanced HF and dyssynchrony ( $\left.\mathrm{HF}_{\mathrm{DYS}}\right)$ with or without subsequent echocardiographic improvement after CRT. We discovered a novel HF-associated miRNA (miR-30d), baseline levels of which were associated with CRT response. We further defined the spatial heterogeneity in expression of miR-30d in a canine model of dyssynchrony, and found that miR-30d is synthesized and released by cardiomyocytes (CM) in response to increased mechanical stress. MiR-30d mediated CM hypertrophy with adaptive features, and was protective against TNF-a-induced CM apoptosis. These findings suggest plasma miR-30d not only has a potential role as a prognostic biomarker, but may also be functionally implicated as an adaptive response to $\mathrm{HF}_{\mathrm{DYS}}$, protecting against deleterious TNF-a signaling. 


\section{METHODS}

\section{Patient population}

52 (12 for initial discovery and 40 for initial validation) patients referred for CRT pacemaker implantation for clinical indications (class II-IV heart failure with LV ejection fraction < 35\% and QRS duration > $120 \mathrm{msec}$ with left bundle branch block were studied). Subjects were limited to patients with clear electrical dyssynchrony (LBBB or RV pacing), who would be expected to respond to CRT. Twenty-one additional subjects, with banked plasma samples at both baseline and 6 months, and who had clear electrical dyssynchrony (LBBB or RV pacing) were obtained from a parallel longitudinal study to assess changes in miRNA levels post-CRT. Clinical, demographic, and echocardiographic characteristics were abstracted from the electronic medical record. Transthoracic echocardiography was performed for clinical indications pre- and post-CRT (Table 1). LV ejection fraction was calculated by either a single dimension method or method of discs at the discretion of the interpreter. Samples of peripheral venous and coronary sinus blood were collected at the time of CRT pacemaker implantation. Plasma from control patients who did not have cardiac disease was obtained from a cohort of 29 patients with non-cardiac dyspnea. RNA was extracted using the mirVANA PARIS RNA isolation kit (Ambion, Inc., Grand Island, $\mathrm{NY}$ ) and samples were promptly frozen at $-80^{\circ} \mathrm{C}$ (with $50 \mathrm{pmol}$ of $C$. Elegans miRNA [celmiR 39] as spike-in control; Ambion). An institutional review committee approved the study, and all subjects gave informed consent for participation in the study.

\section{Discovery of candidate miRNAs}

In the initial discovery analysis, miRNA profiles in 6 patients without change in ejection fraction (mean change in EF 1\%) and 6 patients with a robust improvement in ejection fraction (mean EF increase 24\% at 6 months) were assessed using the Megaplex miRNA primer pool (Applied Biosystems, Foster City, CA). Samples prepared as above were reverse transcribed using a pool of stem-looped primers designed to amplify a total of 766 different miRNAs. The pool of reverse transcribed RNA was subjected to quantitative PCR (qPCR) on human microRNA microarrays containing 383 miRNA species each (ABI, Human MicroRNA microarray A and B cards). The results were analyzed using nonhierarchical clustering to look for miRNAs that differed significantly between the groups, with $\mathrm{p}$ values adjusted using a proprietary Benjamini-Hochberg FDR correction in the DataAssist3.0 software package.

\section{Canine cardiac sample preparation}

The canine model of dyssynchronous heart failure has been previously described. Details of the model, sample extraction and preparation are provided in the Supplementary Materials. The protocols followed were in accordance with institutional guidelines.

\section{Neonatal rat ventricular myocyte transfection, treatment, and fixation}

Neonatal rat ventricular CMs were harvested and prepared from 1 day postnatal SpragueDawley rats as previously described ${ }^{14}$. Details are provided in Supplementary Methods. TNF-a treatment was performed by adding TNF-a Sigma Aldrich) to a final concentration 
of $25 \mathrm{ng} / \mathrm{mL}$ for 24 hours. Cells were harvested by mechanical scraping followed by RNA or protein extraction. Alternatively, an apoptosis assay was performed on TNF-a treated cells using the cell death detection ELISA ${ }^{\text {PLUS }}$ kit (Roche) according to manufacturer's protocol.

\section{Mechanical Stretch}

Mechanical stretch of CMs was performed using previously described apparatus developed by Zhuang et al. ${ }^{15}$. Preparation of exosomes was performed as previously described ${ }^{16}$. For details see Supplemental Methods.

\section{Reverse transcription-quantitative PCR (RT-PCR)}

RT-PCR of individual candidate miRNAs was performed using the TaqMan qRT PCR kit (Invitrogen, Inc.), per the manufacturers instruction. Details of reaction chemistry, quality control for our assay (Fig. S1), primer sequences, and analysis of quantitative PCR data are provided in Supplemental Methods.

\section{Western Blotting}

Samples were separated using SDS-PAGE, transferred to nitrocellulose membrane (Bio Rad) and subjected to immunoblotting as previously described. Details of the antibodies used are provided in the supplemental data.

\section{Differential Gene Expression and Pathway Analysis}

Gene expression arrays were downloaded from NIH-GEO (GSE14661) and normalized by quartile normalization using Bioconductor ${ }^{17}$. Differentially expressed probes were identified by linear model using the LIMMA package ${ }^{18}$. Benjamini-Hochberg method was used to correct $\mathrm{P}$ values for multiple comparison tests. Probes with corrected values of less than 0.01 and log fold-change of more than 1.2 were included in the analysis. The differentially expressed genes were further analyzed for enriched functional pathways using Ingenuity Pathway Analysis (Ingenuity Systems, www.ingenuity.com). Pathways contain genes predicted to be targeted by miR-30d using miRBase ${ }^{19,}{ }^{20}$, TARScan ${ }^{20}$, TarBase ${ }^{21}$ were identified.

\section{Statistical Methods}

Baseline clinical and demographic variables were compared by Student's $t$-test (for normally distributed continuous variables) and Wilcoxon testing (for non-normally distributed continuous variables) or Fisher exact test (for categorical data). The protocol for how candidate miRNA biomarkers were identified is discussed below (see Results, Derivation cohort). We validated these candidate miRNAs in our overall population $(\mathrm{N}=40$; see Results, Validation cohort).

For the purposes of regression analysis, miRNAs levels (relative to spike-in control miR-39) were log-transformed to account for non-linearity. To determine the association between changes in LVEF (our primary outcome) and selected miRNAs, we calculated Spearman correlation coefficients between baseline miR levels and change in LVEF (baseline to follow-up). Candidate miRNAs from our derivation cohort (30d, 29c, 766, 142-5p) were 
entered into a univariate logistic regression model to determine the unadjusted association between miR level and outcome ("CRT responder" versus "CRT non-responder"). MiRs significant in univariate logistic regression were (1) entered into logistic models adjusted for covariates important in CRT responsiveness (pre-implant QRS, LVEF, prior revascularization, and serum creatinine) and (2) tested for correlation with absolute change in LVEF (pre- vs. post-CRT). A CRT "responder" was defined as an increase in LV ejection fraction $\geq 10 \%$ between baseline and follow-up transthoracic echocardiography after CRT pacemaker implantation. We used standard Youden index analysis (MedCalc v12.5.0) to calculate optimal cut-point and sensitivity and specificity for log-transformed miR-30d for CRT response.

We also checked whether the relationship between miR-30d and change in LVEF is significantly different for the two cohorts of 40 and 21 patients using a dummy variable to specify the cohort. We used logistic regression to test whether the association of miR-30d with response to CRT (by LVEF), was modified by cohort (using an interaction term between the dummy variable and miR-30d). The interaction term was non-significant (while miR-30d remained significant), suggesting no effect modification by cohort.

For miRNA analysis, $\mathrm{C}_{\mathrm{t}}$ values from responder, non-responder, and volunteer (non-HF) population were compared to the level of the spike-in C. Elegans miR-39 to generate a " $\Delta \mathrm{C}_{\mathrm{t}}$ value". When tissue levels of miRNAs were measured, a nucleolar RNA (U6 snRNA) was used as an internal reference and control for tissue extraction. The exponentiated $\Delta \mathrm{C}_{\mathrm{t}}$ (i.e. $2^{-\Delta \mathrm{Ct}}$ ) represents a concentration relative to the spike-in or endogenous miRNA, as has been previously described. To determine the relative fold difference between respective groups, the exponentiated $\Delta \mathrm{C}_{\mathrm{t}}$ values between responders and non-responders were compared (base 2) to generate fold change.

Differences in miRNA concentrations between groups (volunteer vs. non-responder; volunteer vs. responder) were evaluated using an unpaired Student's $t$-test of average CT values in each group. For comparisons of non-normally distributed data (as assessed by Shapiro-Wilk testing), a Mann-Whitney test was used for comparison. Paired T test was used when appropriate (i.e. when comparing coronary sinus and peripheral plasma in the same patient). Benjamini Hochberg 22 (for False Discovery Rate of 5\%) was used for correction of multiple hypothesis testing in the validation cohort.

A two-sided $\mathrm{P}$ value $<0.05$ was considered significant for all analyses. All statistical analyses were performed using SAS 9.3 (SAS Institute, Cary, NC) or Microsoft Excel.

\section{RESULTS}

\section{Discovery of plasma miRNAs signature of CRT response}

From our cohort of patients with $\mathrm{HF}_{\mathrm{DYS}}$ who were referred for CRT implantation, we identified 6 with the greatest subsequent rise in ejection fraction $(\mathrm{EF})$ and 6 with no change (or decline) in EF measured 6 months after CRT was initiated, as our discovery cohort (Table S1). To improve the sensitivity of our screen and minimize confounding variables, we restricted this discovery cohort to male patients with non-ischemic cardiomyopathies and 
clear dyssynchrony as manifested by left bundle branch block. There were no significant differences between baseline age, gender, NYHA class and LVEF between the two groups in this discovery cohort. From analysis of 766 known human miRNA species in plasma, we identified four miRNAs (miR-409-3p, miR-30d, miR-99b, and miR-766) that were differentially expressed in plasma between the responder and non-responder groups ( $\mathrm{p}<0.05$, $5 \%$ false discovery rate); a fifth miRNA (miR-142-5p) had borderline differences $(\mathrm{p}=0.0582)$. These data have been deposited into the NIH-GEO database.

\section{Validation of prognostic plasma miRNA biomarkers for CRT response}

We validated the findings from the discovery cohort in an additional 40 patients with $\mathrm{HF}_{\mathrm{DYS}}$ who met guideline criteria for CRT, had LBBB (intrinsic or RV-paced) with available baseline plasma that passed quality control for isolated ex-RNA (lack of hemolysis and significant DNA contamination). Baseline (pre-CRT) levels of the plasma miRNAs identified in our discovery cohort were analyzed in this prospective cohort that were followed for at least 6 months following their CRT implantation with serial echocardiograms. CRT response was defined by an increase in LVEF $\geq 10 \% 6$ months after implant. Patient characteristics of this cohort, stratified by CRT responder $(\mathrm{N}=21 ; 53 \%)$ or non-responder $(\mathrm{N}=19 ; 48 \%)$ status are shown in Table 1. The average LVEF, LV dimensions and volumes as well as QRS duration before CRT implantation were not significantly different between the two groups. There was a trend towards more prior revascularization in non-responders (68\% vs. $33 \%$; $\mathrm{P}=0.06$ ), but pre-implant disease severity and medication use was similar. At 6 months after CRT, responders had a significant increase in LVEF compared with non-responders ( $41 \pm 9 \%$ versus $28 \pm 7 \%$ respectively). For those patients whose $\mathrm{LV}$ volumes were available, the CRT responders also had evidence of reverse LV remodeling with a significant decrease in LV end-systolic volume Fig. 1A, Table 1).

Of the five miRNAs identified in our derivation cohort (409-3p, 30d, 99b, 766 and 142-5p), only miR-30d, miR-766, and miR-142-5p were significantly different between the two groups in the 40 patient validation cohort ( $\mathrm{p}<0.05$ by Wilcoxon with Benjamini-Hochberg correction) (Figure 1B). Because arrays on plasma ex-RNAs may lack sensitivity for discovery due to very low amounts of total RNA and presence of inhibitors in plasma, we additionally measured levels of miRNAs (miR-129-5p, miR-18b, miR-622, miR-423-5p and miR-29c), previously described as differentially expressed in HF compared with healthy controls $^{23}$. Of these HF-associated miRs, only miR-29c was significantly different between responders and non-responders (Figure 1C) in the validation cohort. Finally, to determine the plasma levels of these miRs in non-HF patients, we assessed their levels in a 'control cohort' of 29 patients with dyspnea referred for cardiopulmonary exercise testing and found not to have any cardiovascular disease (Table 2). Levels of miR-30d, miR-142-5p and miR-29c species were significantly elevated in the HF patients (both responders and nonresponders) compared to the control cohort, while miR-766 was similar in control patients and responders, but significantly decreased in CRT non-responders (Figure 1D-G). 


\section{Predictors of CRT responsiveness}

We used univariate logistic regression to determine covariates that were associated with a positive response to CRT as defined above. Only the presence of prior revascularization was associated with a lower probability of CRT response ( $\mathrm{OR}=0.23,95 \%$ CI $0.06-0.87, \mathrm{p}=0.03$; Table 3, and Forest plot in Fig. 2A). Of the candidate miRNAs that were differentially expressed between responders and non-responders in the validation cohort, only miR-30d, miR-766, and miR-142-5p were associated with a greater likelihood of CRT response.

To further assess the relationship between miRNA levels and improvement in LV function, we built multivariable regression models (Table S2) for each of the miRNAs significant in the univariate logistic regression (Table 3). In these models, we included clinical variables significantly associated with response to CRT in the univariate regression (Table 3) in addition to variables important in CRT responsiveness (e.g., serum creatinine, prior revascularization, pre-implant QRS duration, LVEF). When adjusted for these covariates, miR-30d (HR 2.52, CI 1.07-5.94) and miR-142-5p (HR 2.47, CI 1.26-4.85) were associated with CRT responsiveness, while miR-766 was not. To address which miRNA biomarker was more strongly associated with change in LVEF in our dataset, we entered both miR-142-5p and miR-30d (log-transformed) into a best-overall multivariable linear model (containing serum creatinine, prior revascularization, pre-implant QRS duration, LVEF). In this model, only log-transformed miR-30d $(\beta=2.69, \mathrm{p}=0.02)$ was associated with change in LVEF. Interestingly, there was excellent correlation between baseline miR-30d and miR-142-5p levels raising the possibility of co-regulation of these miRs (Fig. S2).

Although age and gender were not correlated with CRT response in this data set (Table 3), given the importance of age and gender on miRNA levels ${ }^{24}$, we also ensured that miR-30d remained significantly associated with CRT response after adjustment for age and gender $(\mathrm{OR}=3.1$ per $\log , \mathrm{CI}=1.33-7.26, \mathrm{p}=0.009$, Table $\mathrm{S} 3)$.

MiR-30d (Spearman $\rho=0.39, p=0.01$ ) was significantly correlated with change in LVEF at 6 months after CRT (Fig. 2B), and we found a strong correlation between baseline miR-30d levels and probability of a favorable CRT response (increase in LVEF $\geq 10 \%$, Fig. 2C) using the estimated parameters of logistic regression of miR-30d level on changes in LVEF. Removal of the two patients with the highest miRNA levels (Figure 2B) resulted in a near significant association of miRNA levels and change in $\mathrm{EF}$ (Spearman $\rho=0.31, p=0.058$ ), while the univariable logistic association between log-transformed miR-30d and CRT response remained significant (OR 2.24, 95\% CI 1.08-4.67; $\mathrm{P}=0.03$ ) even after removal of these extreme values.

In an ROC analysis, miR-30d was a significant discriminator of CRT responsiveness (Cstatistic $0.744,95 \% \mathrm{CI}=0.589-0.899$, with sensitivity and specificity for the Youden optimal cut point for miR-30d $57.1 \%$ and $89.5 \%$, respectively) while baseline QRS duration, a known clinical predictor for CRT response ${ }^{6,25}$, did not predict response in our cohort (Cstatistic 0.542, CI=0.354-0.731, Fig. 2D).

In order to determine the change in miR-30d levels over time after CRT placement, we measured miR-30d levels in 21 additional patients from a longitudinal prospective cohort 
who had plasma collected both at baseline and 6 months after CRT. When combined with the initial 40 patients validation cohort the relationship between miR-30d and changes in LVEF ( $p=0.02$, Fig. S3) remained in the 61 patients, supporting the statistical robustness of this relationship. This relationship holds for the combined cohort irrespective of whether the samples are derived from the original cohort of 40 patients or the newer cohort of 21 patients (see Methods for details of this analysis) further validating our initial observation.

Comparing the change in miR-30d over time in this cohort, we found that miR-30d levels decreased in responders at the 6 month time point $(\mathrm{p}=0.05)$, while remaining unchanged in the non-responders (Fig. 2E), suggesting that miR-30d may be dynamically regulated in response to resynchronization in those patients who respond and presumably reduce ventricular wall stress through reverse remodeling.

Given that plasma miR-30d was the most promising biomarker correlated with changes in $\mathrm{LV}$ function post-CRT, we next examine the biological significance of miR-30d in myocardial remodeling in the context of $\mathrm{HF}_{\mathrm{DY}}$.

\section{Origin and regulation of plasma miR-30d}

Levels of plasma miR-30d were 18 -fold higher in coronary sinus (CS) plasma compared with contemporaneous peripheral plasma samples taken at the time of CRT implantation (n $=6$ paired samples, $\mathrm{p}=0.011$ by paired T-test, see Fig. $3 \mathrm{~A}$ ), suggesting a cardiac origin for plasma miR-30d. We next assessed the cell type that was the possible source of the extracellular miR-30d and found that miR-30d was expressed at 5-fold higher level in cardiomyocytes (CM) relative to cardiac fibroblasts (Fig. 3B) derived from neonatal rat ventricles (see Fig. S4 for validation of cell culture purity). Because extracellular RNAs may be packaged in extracellular vesicles (EVs), we assessed the content of CM-derived EVs (isolated by FACS) and found a marked enrichment of miR-30d (Fig. 3C). Taken together, these data suggest that CMs release miR-30d packaged in EVs.

To determine how miR-30d is regulated in the heart in vivo, we assessed levels of miR-30d in tissue samples derived from a canine model of $\mathrm{HF}_{\mathrm{DYS}}$ and CRT ${ }^{26}$. The $\mathrm{HF}_{\mathrm{DYS}}$ dogs respond to CRT favorably, hence providing a model that allows for assessing dynamic changes in miR-30d. We found that miR-30d (normalized to U6, see Fig. S5) was elevated in both $\mathrm{HF}_{\mathrm{DYS}}$ and CRT dogs relative to control dogs (Fig. 3D). Of note, miR-30d levels were highest in the lateral wall of $\mathrm{HF}_{\mathrm{DYS}} \operatorname{dogs}(\mathrm{p}<0.05$ versus septal wall by paired $\mathrm{t}$-test) corresponding to the area of highest wall stress. This heterogeneity in expression between the septal and lateral wall was eliminated following resynchronization in CRT animals. These data suggest that in this canine model (similar to responder patients), miR-30d is upregulated by the increased wall stress associated with dyssynchrony, but decreases with the concomitant decrease in wall stress following resynchronization. Interestingly, similar to miR-30d, miR-142-5p levels were highest in the lateral wall of $\mathrm{HF}_{\mathrm{DYS}}$, and decreased with CRT in the canine model (Fig. S6).

To better understand the mechanism of miR-30d regulation, we assessed miR-30d (both extracellular and intracellular) in an in vitro model of mechanical stress (neonatal CMs seeded on a silicone membrane exposed to cyclic stretch at $3 \mathrm{~Hz}$ ) ${ }^{27}$. We found an increase 
in EV-containing miR-30d following stretch, suggesting stress-induced release of miR-30d by CMs both acutely at 6 hours (20\% stretch) (Fig. 3E) and after longer duration of stretch (24 hours at 10\%, Fig. 3F). In the acute model, intracellular miR-30d levels were not significantly changed, suggesting replacement of released EV-containing miR-30d by newly synthesized miR-30d, while longer duration of stretch was associated with an increase in the cellular levels of miR-30d.

\section{Functional role of miR-30d}

With the recent discovery that extracellular RNAs may be implicated in disease pathogenesis ${ }^{9}$, we sought to investigate the possible functions of miR-30d in CMs. Overexpression of miR-30d (Fig. S7) via transient transfection of miR-30d (miRNA mimic, Invitrogen, Inc) into CMs induced cellular growth as assessed by quantification of CM size (Fig. 4A). While cell size clearly increased with miR-30d overexpression, there was no significant change in the mRNA levels of several genes associated with pathological hypertrophy and fibrosis: notably ANP, $\alpha / \beta-M H C$ ratio, and CTGF did not change, although BNP increased 48 hours after transfection with miR-30d mimic (Fig. 4B).

To assess the effect of miR-30d on signaling pathways implicated in cardiac hypertrophy, lysates from CMs transfected with miR-30d mimic or a scrambled control, were subjected to immunoblotting with antibodies against key signaling molecules. After 48 hours of miR-30d expression, there was an increase in phosphorylated Akt1 (Ser-473), a serine-threonine kinase that is necessary and sufficient for 'physiological hypertrophy' ${ }^{28,29}$, but not p38 and ERK1/2, the signaling effectors implicated in pathological cardiac hypertrophy (Fig. 4C). While there are undoubtedly complex interactions between these pathways, our results suggest that overexpression of miR-30d in CMs mediates CM growth with molecular features most consistent with adaptive hypertrophy.

To further explore the functional role of miR-30d, we identified miR-30d targets that are dynamically regulated in the canine model of $\mathrm{HF}_{\mathrm{DYS}}$. Using a LIMMA (linear models for microarray data) approach, genes whose expression was inversely correlated to miR-30d expression (i.e. genes which were down-regulated in the lateral wall of dyssynchronous dogs where miR-30d levels were highest compared to the septal wall) ${ }^{30}$ were identified and screened for putative miR-30d targets using the Tarbase and TargetScan prediction algorithms ${ }^{20,31}$. Subsequently, using the IPA (Ingenuity Pathways Analysis) approach we generated predicted functional pathways associated with the observed changes in miR-30d expression (see schematic in Fig. 5A). Several of these genes affect multiple biologically relevant pathways in the heart, specifically, LIMS1, PPP1R14c, MAK3K13, and JAK1 (Fig. 5B). In addition, several other miR-30d targets with known roles in cardiac hypertrophy were identified using Tarbase. Intriguingly, one of these, mitogen associated protein kinase 4 (MAP4K4), a downstream mediator of tumor necrosis factor-alpha (TNF- $\alpha$ ) has recently been identified as a target of miR-30d in pancreatic tissue ${ }^{32}$ and has been shown to play a key role in TNF-mediated inflammatory processes. Because TNF-a not only plays an important role in pathogenesis of cardiac remodeling, but also is differentially expressed in the lateral and septal walls in the $\mathrm{HF}_{\mathrm{DYS}}$ canine model ${ }^{33}$, we deemed MAP4K4 a potentially important target of miR-30d in $\mathrm{HF}_{\mathrm{DYS}}$. 
To validate candidates identified through bioinformatics analyses, we assessed the ability of miR-30d overexpression in CMs to effectively silence the mRNAs for the putative targets. Transient transfection of CMs with miR-30d caused a significant decrease in the mRNA levels of LIMS1, MAP4K4, and PPP1R14c (Fig. 5C) relative to scramble transfected cells. Given the involvement of TNF-a signaling in the pathophysiology of $\mathrm{HF}_{\mathrm{DYS}}$ as described above, we focused on the interaction between miR-30d and MAP4K4. MAP4K4 protein was significantly decreased (Fig. 5D) in the lateral wall in $\mathrm{HF}_{\mathrm{DYS}}$ compared to lateral wall of control animals, correlating inversely with miR-30d levels in these two regions. Conversely, in the CRT lateral wall (where wall stress presumably decreased with resynchronization), miR-30d levels fell compared to $\mathrm{HF}_{\mathrm{DYS}}$, and MAP4K4 protein level returned to control levels, suggesting that MAP4K4 was an in vivo target of miR-30d in these models.

We next investigated the functional role of miR-30d in CM biology. Treatment of scramble transfected CMs with TNF-a led to a robust increase in MAP4K4 mRNA, which was markedly attenuated by miR-30d overexpression (Fig. 6A). TNF-a is an important mediator of $\mathrm{CM}$ apoptosis in HF; hence we sought to determine if miR-30d could modulate the effects of TNF- $a$ on CMs. TNF- $a$ caused a marked increase in apoptosis in scrambled controltransfected CMs as determined by an assay for DNA fragmentation (Fig. 6A, right panel). MiR-30d transfection inhibited TNF-a-mediated apoptosis in CMs, suggesting that miR-30d may be protective against the maladaptive effects of TNF-a. Finally, miR-30d transfection also prevented TNF-a-mediated increase in molecular markers associated with pathological hypertrophy and fibrosis $\beta$-MHC, Fig. 6C). Together these data suggested that miR-30d may counteract TNF-a mediated adverse processes in CMs either directly via down-regulation of MAP4K4 or through other indirect pathways. To gather preliminary insight into the protective effect of miR-30d in human patients, we measured levels of high sensitivity Troponin $\mathrm{T}$, a marker of myocardial injury in our patient cohorts. Interestingly, miR-30d levels were inversely correlated with levels of hs-TnT (Spearman $\mathrm{r}=-0.51, \mathrm{p}=0.001$, Fig. $6 \mathrm{C}$ ), suggesting that higher levels of miR-30d may be cardioprotective in human HF patients.

These experiments provide 'in vivo' supportive evidence for our hypothesis that miR-30d may be protective against inflammation (TNF-a) induced cardiomyocyte injury, thereby promoting cardiomyocyte survival and favorably influencing the degree of cardiac remodeling in response to CRT.

\section{DISCUSSION}

In this study, we describe the discovery and characterization of a novel circulating extracellular miRNA implicated in the response to CRT in patients with $\mathrm{HF}_{\mathrm{DYs}}$. In a cohort of patients with $\mathrm{HF}_{\mathrm{DYS}}$, miR-30d was associated with a favorable CRT response as defined by an improvement in LV function (increase in LVEF $>10 \%$ ) as well as with LV remodeling (decrease in LVESV). MiR-30d was independently associated with CRT response, with discriminative ability superior to currently used clinical variables such as QRS duration. Based on this initial clinical finding, we characterized its functional role further. We found that miR-30d is enriched in the coronary sinus of patients with HF, elevated in myocardial tissue in canine $\mathrm{HF}_{\mathrm{DYS}}$ with higher expression in $\mathrm{CMs}$ relative to 
cardiac fibroblasts, collectively suggesting a cardiac origin of circulating miR-30d. MiR-30d appears to be regulated by wall stress, consistent with its increased expression in the lateral wall in the canine model of $\mathrm{HF}_{\mathrm{DYS}}$, and is dynamically regulated in response to CRT. Finally, miR-30d appears to play a functional role in CM biology, mediating cardiac hypertrophy with a distinct molecular signature, and protection against TNF-a-induced apoptosis and deleterious signaling in vitro. More generally, our study supports an emerging paradigm that circulating plasma miRNAs serve not only as reporters of disease severity, but may also play a functional role in the pathogenesis of human disease.

Clinically, the use of biomarkers (imaging and serologic) to predict response to CRT has been a critical goal in modern electrophysiology research. Greater than $30 \%$ of patients selected for CRT based on current guidelines fail to demonstrate benefit, and other metrics of predicting successful response to CRT (including echocardiographic parameters ${ }^{34}$, multimarker clinical scores ${ }^{35}$ or protein biomarkers ${ }^{8,35}$ ) to define patients likely to respond to CRT have not successfully lowered this the non-response rate. In this study, we demonstrated that miR-30d (a novel extracellular RNA biomarker) is associated with response to CRT independent of clinical markers of risk. If validated in larger populations, assessment of miR-30d levels in patients with $\mathrm{HF}_{\mathrm{DYS}}$ could constitute a major step forward in HF disease management and patient selection for CRT.

The results of this study should be viewed in the context of its design. Human sample size was not sufficient to yield $80 \%$ power except for large effects, or to provide adequate precision for odds ratio estimates. Nevertheless, we observed a novel functional role for miR-30d in cardiac remodeling, and observed consistent associations between miR-30d and LVEF, robust to adjustment for important clinical variables. The findings from this initial study can inform future studies to validate the prognostic role of plasma miR-30d in larger and more diverse cohorts. Due to the limitations of available data, we used LVEF rather than volumes to assess CRT response in this study, but notably in subjects where volume assessment was possible, responders classified by LVEF also showed reverse remodeling as assessed by volumes (Fig. S1). While there was evidence of parallel regulation of miR-142-5p with CRT response in our clinical pilot study, a multi-marker signature that includes multiple miRNAs, protein biomarkers and clinical variables will require a larger cohort for adequate statistical power. Our cohort did not have sufficient clinical events (e.g. mortality and HF hospitalizations) to determine if miR-30d can also predict 'clinical' response to CRT, and larger studies with longer duration of follow-up will be needed answer these important questions in the future. Finally, several scientific questions, including the optimal method of measurement of plasma miRNAs, techniques of normalization, and reference profiles in healthy individuals need to be determined prior to adoption of these novel biomarkers into the clinic.

Our results support the hypothesis established by recent studies that clinically useful extracellular RNA biomarkers may be functionally implicated in disease pathogenesis ${ }^{12}$. Mir-30d appears to be dynamically regulated by mechanical stretch and released in EVs by CMs. While the miR-30 family has been previously implicated in CM hypertrophy, our results extend these studies in important new directions and suggest that it may have a protective effect in CMs. We hypothesize that miR-30d may in fact constitute an adaptive 
response that is induced by increased wall stress in $\mathrm{HF}_{\mathrm{DYS}}$. In turn, miR-30d ameliorates the effects of other deleterious processes that are also induced by abnormal wall stress, such as TNF-a signaling and downstream signaling cascades leading to cell death (Fig. 6D). Therefore, higher miR-30d levels, as seen in the human 'responder' patients or the canine $\mathrm{HF}_{\mathrm{DYS}}$ models, may preserve myocardial viability and the capacity for beneficial $\mathrm{LV}$ remodeling. The lower levels of miR-30d in "non-responders" may reflect a cohort that are either unable to mount a sufficient miR-30d response, or have reached a 'burnt out' stage in the progression of their disease. In either case, the lack of an adequate miR-30d response to counteract TNF signaling may lead to CM death and adverse remodeling. Among CRT responders (and canines receiving CRT), as wall stress decreases with CRT, both the maladaptive pathways and therefore the need for protective miR-30d signaling would decrease, corresponding to lower miR-30d levels in responder patients or in the canine CRT model.

We recognize our mechanistic insights rely on cultured CMs derived from neonatal rat ventricles, which are an imperfect model of adult CM biology, but one that has been extensively used and validated for the study of signaling pathways. Secondly, EV-containing miR-30d may have a role in paracrine signaling ${ }^{16}$ with effects on other cell types that may ultimately determine the organ-level effect of plasma miR-30d. Finally, we relied on a bioinformatics approach for initial identification of miR-30d targets, which can introduce bias (and therefore was complemented with validation in $\mathrm{CMs}$ ). However future detailed analysis of the RISCome may identify other relevant targets of importance. Definitive proof of whether miR-30d is beneficial, and its mechanism in vivo can only be obtained with gain or loss-of-function experiments in animal models of $\mathrm{HF}_{\mathrm{DYS}}$, which are beyond the scope of this study.

\section{Supplementary Material}

Refer to Web version on PubMed Central for supplementary material.

\section{Acknowledgments}

Funding Sources: SD is funded by the Klarman Scholars foundation and a grant from NIH Common Funds UH2TR000901 through the Office of Strategic Coordination/Office of the NIH director.

\section{References}

1. Kass DA. An epidemic of dyssynchrony: But what does it mean? J Am Coll Cardiol. 2008; 51:1217. [PubMed: 18174030]

2. Vaillant C, Martins RP, Donal E, Leclercq C, Thebault C, Behar N, Mabo P, Daubert JC. Resolution of left bundle branch block-induced cardiomyopathy by cardiac resynchronization therapy. J Am Coll Cardiol. 2013; 61:1089-1095. [PubMed: 23352778]

3. Kass DA. Ventricular resynchronization: Pathophysiology and identification of responders. Rev Cardiovasc Med. 2003; 4(Suppl 2):S3-S13. [PubMed: 12776008]

4. Goldenberg I, Hall WJ, Beck CA, Moss AJ, Barsheshet A, McNitt S, Polonsky S, Brown MW, Zareba W. Reduction of the risk of recurring heart failure events with cardiac resynchronization therapy: Madit-crt (multicenter automatic defibrillator implantation trial with cardiac resynchronization therapy). J Am Coll Cardiol. 2011; 58:729-737. [PubMed: 21816309] 
5. Barsheshet A, Goldenberg I, Moss AJ, Eldar M, Huang DT, McNitt S, Klein HU, Hall WJ, Brown MW, Goldberger JJ, Goldstein RE, Schuger C, Zareba W, Daubert JP. Response to preventive cardiac resynchronization therapy in patients with ischaemic and nonischaemic cardiomyopathy in madit-crt. Eur Heart J. 2011; 32:1622-1630. [PubMed: 21075774]

6. Peterson PN, Greiner MA, Qualls LG, Al-Khatib SM, Curtis JP, Fonarow GC, Hammill SC, Heidenreich PA, Hammill BG, Piccini JP, Hernandez AF, Curtis LH, Masoudi FA. Qrs duration, bundle-branch block morphology, and outcomes among older patients with heart failure receiving cardiac resynchronization therapy. Jama. 2013; 310:617-626. [PubMed: 23942680]

7. Muto C, Gasparini M, Iacopino S, Peraldo C, Curnis A, Sassone B, Diotallevi P, Davinelli M, Valsecchi S, Tuccillo B. Efficacy of low-dose dobutamine stress-echocardiography to predict cardiac resynchronization therapy response (lodo-crt) multicenter prospective study: Design and rationale. Am Heart J. 2008; 156:656-661. [PubMed: 18926147]

8. Lopez-Andres N, Rossignol P, Iraqi W, Fay R, Nuee J, Ghio S, Cleland JG, Zannad F, Lacolley P. Association of galectin-3 and fibrosis markers with long-term cardiovascular outcomes in patients with heart failure, left ventricular dysfunction, and dyssynchrony: Insights from the care-hf (cardiac resynchronization in heart failure) trial. Eur J Heart Fail. 2012; 14:74-81. [PubMed: 22089058]

9. Zampetaki A, Mayr M. Micrornas in vascular and metabolic disease. Circ Res. 2012; 110:508-522. [PubMed: 22302757]

10. Montgomery RL, Hullinger TG, Semus HM, Dickinson BA, Seto AG, Lynch JM, Stack C, Latimer PA, Olson EN, van Rooij E. Therapeutic inhibition of mir-208a improves cardiac function and survival during heart failure. Circulation. 2011; 124:1537-1547. [PubMed: 21900086]

11. Ganesan J, Ramanujam D, Sassi Y, Ahles A, Jentzsch C, Werfel S, Leierseder S, Loyer X, Giacca M, Zentilin L, Thum T, Laggerbauer B, Engelhardt S. Mir-378 controls cardiac hypertrophy by combined repression of mitogen-activated protein kinase pathway factors. Circulation. 2013; 127:2097-2106. [PubMed: 23625957]

12. Creemers EE, Tijsen AJ, Pinto YM. Circulating micrornas: Novel biomarkers and extracellular communicators in cardiovascular disease? Circ Res. 2012; 110:483-495. [PubMed: 22302755]

13. Zhu H, Fan GC. Extracellular/circulating micrornas and their potential role in cardiovascular disease. Am J Cardiovasc Dis. 2011; 1:138-149. [PubMed: 22059153]

14. Das SAT, Rosenberg M, Hessler K, Xiao C, Quintero PA, Ottaviano FG, Knight AC, Graham EL, Boström P, Morissette MR, del Monte F, Begley MJ, Cantley LC, Ellinor PT, Tomaselli GF, Rosenzweig A. Pathological role of serum- and glucocorticoid-regulated kinase 1 in adverse ventricular remodeling. Circulation. 2012; 126:2208-2219. [PubMed: 23019294]

15. Zhuang J, Yamada KA, Saffitz JE, Kleber AG. Pulsatile stretch remodels cell-to-cell communication in cultured myocytes. Circ Res. 2000; 87:316-322. [PubMed: 10948066]

16. Waldenstrom A, Genneback N, Hellman U, Ronquist G. Cardiomyocyte microvesicles contain DNA/rna and convey biological messages to target cells. PLoS One. 2012; 7:e34653. [PubMed: 22506041]

17. Taminau J, Steenhoff D, Coletta A, Meganck S, Lazar C, de Schaetzen V, Duque R, Molter C, Bersini H, Nowe A, Weiss Solis DY. Insilicodb: An r/bioconductor package for accessing human affymetrix expert-curated datasets from geo. Bioinformatics. 2011; 27:3204-3205. [PubMed: 21937664]

18. Wettenhall JM, Smyth GK. Limmagui: A graphical user interface for linear modeling of microarray data. Bioinformatics. 2004; 20:3705-3706. [PubMed: 15297296]

19. Griffiths-Jones S. The microrna registry. Nucleic Acids Res. 2004; 32:D109-111. [PubMed: 14681370]

20. Lewis BP, Burge CB, Bartel DP. Conserved seed pairing, often flanked by adenosines, indicates that thousands of human genes are microrna targets. Cell. 2005; 120:15-20. [PubMed: 15652477]

21. Papadopoulos GL, Reczko M, Simossis VA, Sethupathy P, Hatzigeorgiou AG. The database of experimentally supported targets: A functional update of tarbase. Nucleic Acids Res. 2009; 37:D155-158. [PubMed: 18957447]

22. Hochberg Y, Benjamini Y. More powerful procedures for multiple significance testing. Stat Med. 1990; 9:811-818. [PubMed: 2218183] 
23. Tijsen AJ, Pinto YM, Creemers EE. Circulating micrornas as diagnostic biomarkers for cardiovascular diseases. Am J Physiol Heart Circ Physiol. 2012; 303:H1085-1095. [PubMed: 22942181]

24. Wang YT, Tsai PC, Liao YC, Hsu CY, Juo SH. Circulating micrornas have a sex-specific association with metabolic syndrome. J Biomed Sci. 2013; 20:72. [PubMed: 24093444]

25. Dupont M, Rickard J, Baranowski B, Varma N, Dresing T, Gabi A, Finucan M, Mullens W, Wilkoff BL, Tang WH. Differential response to cardiac resynchronization therapy and clinical outcomes according to qrs morphology and qrs duration. J Am Coll Cardiol. 2012; 60:592-598. [PubMed: 22796255]

26. Spragg DD, Leclercq C, Loghmani M, Faris OP, Tunin RS, DiSilvestre D, McVeigh ER, Tomaselli GF, Kass DA. Regional alterations in protein expression in the dyssynchronous failing heart. Circulation. 2003; 108:929-932. [PubMed: 12925451]

27. Sadoshima J, Izumo S. Mechanical stretch rapidly activates multiple signal transduction pathways in cardiac myocytes: Potential involvement of an autocrine/paracrine mechanism. Embo J. 1993; 12:1681-1692. [PubMed: 8385610]

28. DeBosch B, Treskov I, Lupu TS, Weinheimer C, Kovacs A, Courtois M, Muslin AJ. Akt1 is required for physiological cardiac growth. Circulation. 2006; 113:2097-2104. [PubMed: 16636172]

29. Matsui T, Li L, Wu JC, Cook SA, Nagoshi T, Picard M, Liao R, Rosenzweig A. Phenotypic spectrum caused by transgenic overexpression of activated akt in the heart. J Biol Chem. 2002; 277:22896-22901. [PubMed: 11943770]

30. Barth AS, Aiba T, Halperin V, DiSilvestre D, Chakir K, Colantuoni C, Tunin RS, Dimaano VL, $\mathrm{Yu}$ W, Abraham TP, Kass DA, Tomaselli GF. Cardiac resynchronization therapy corrects dyssynchrony-induced regional gene expression changes on a genomic level. Circ Cardiovasc Genet. 2009; 2:371-378. [PubMed: 20031609]

31. Vergoulis T, Vlachos IS, Alexiou P, Georgakilas G, Maragkakis M, Reczko M, Gerangelos S, Koziris N, Dalamagas T, Hatzigeorgiou AG. Tarbase 6.0: Capturing the exponential growth of mirna targets with experimental support. Nucleic Acids Res. 2012; 40:D222-229. [PubMed: 22135297]

32. Tang X, Muniappan L, Tang G, Ozcan S. Identification of glucose-regulated mirnas from pancreatic $\{$ beta\} cells reveals a role for mir-30d in insulin transcription. Rna. 2009; 15:287-293. [PubMed: 19096044]

33. Chakir K, Daya SK, Tunin RS, Helm RH, Byrne MJ, Dimaano VL, Lardo AC, Abraham TP, Tomaselli GF, Kass DA. Reversal of global apoptosis and regional stress kinase activation by cardiac resynchronization. Circulation. 2008; 117:1369-1377. [PubMed: 18316490]

34. Khan FZ, Virdee MS, Palmer CR, Pugh PJ, O’Halloran D, Elsik M, Read PA, Begley D, Fynn SP, Dutka DP. Targeted left ventricular lead placement to guide cardiac resynchronization therapy: The target study: A randomized, controlled trial. J Am Coll Cardiol. 2012; 59:1509-1518. [PubMed: 22405632]

35. Heist EK, Taub C, Fan D, Arzola-Castaner D, Alabiad CR, Reddy VY, Mansour M, Mela T, Picard MH, Ruskin JN, Singh JP. Usefulness of a novel "response score" to predict hemodynamic and clinical outcome from cardiac resynchronization therapy. Am J Cardiol. 2006; 97:1732-1736. [PubMed: 16765123] 

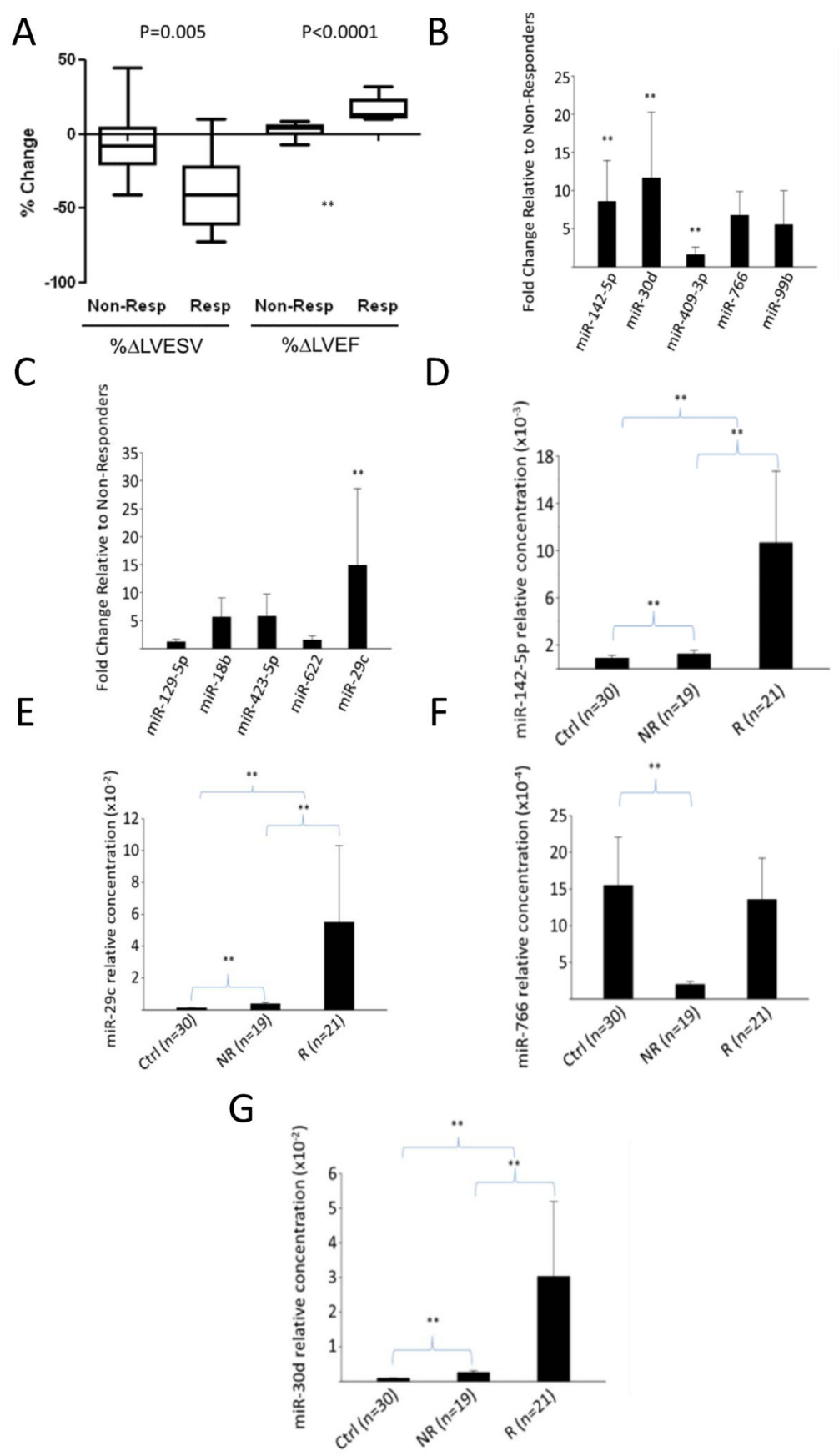

Figure 1.

Differential expression of miRNAs between CRT responders and non-responders in validation cohort. A. Box plot of change in LVESV or LVEF from baseline to follow-up study as measured by available volumetric data ( $\mathrm{N}=29$ ). (LVEF measured in same 29 patients with standard two-dimensional or visual analysis.) $\mathrm{P}$ values reflect Wilcoxon testing. B. miR 142-5p, miR-30d, and miR-766 are significantly increased in responders versus non-responders. C Only miR-29c was significantly increased in responders relative to non-responders. ${ }^{* *}$ denotes $\mathrm{p}<0.05$, Benjamini Hochberg correction for multiple hypothesis 
testing. D-G, Levels of miRNAs identified in A-B in responders, non-responder or control patients. miRNA levels are in arbitrary units, normalized to spike-in C. Elegans miR-39. Ctrl: control patients, NR: non-responders, R: Responders. 


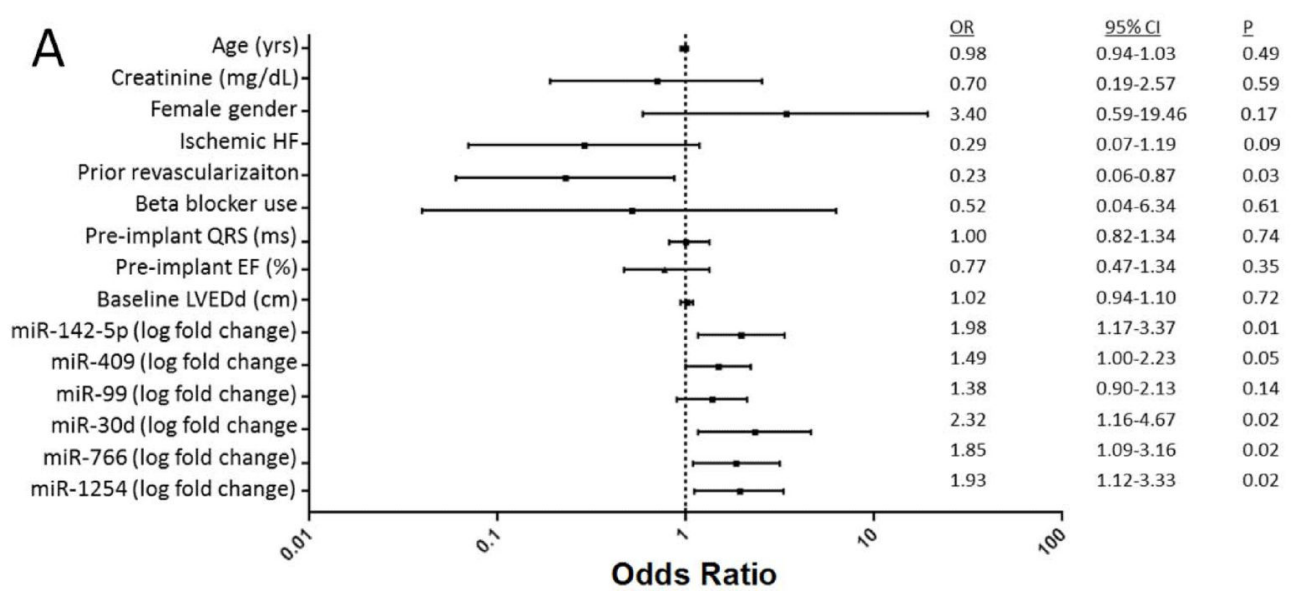

B

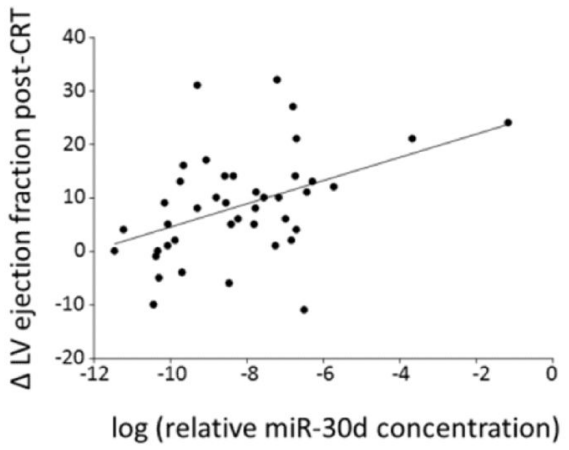

$\mathrm{D}$

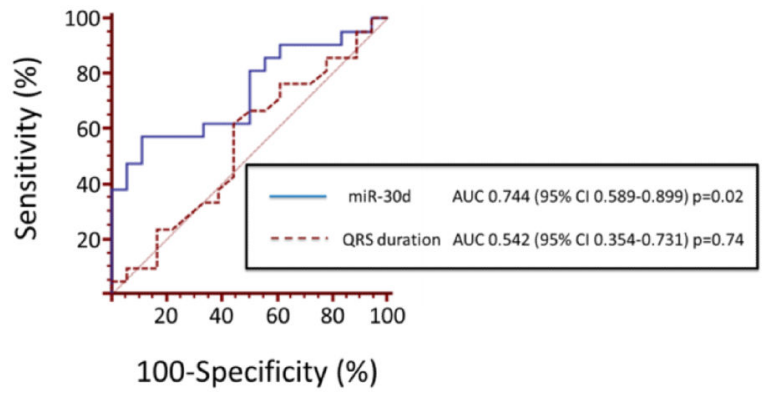

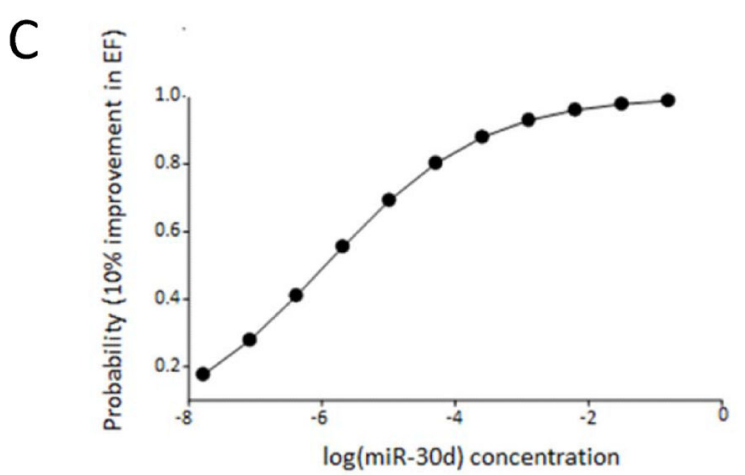

E

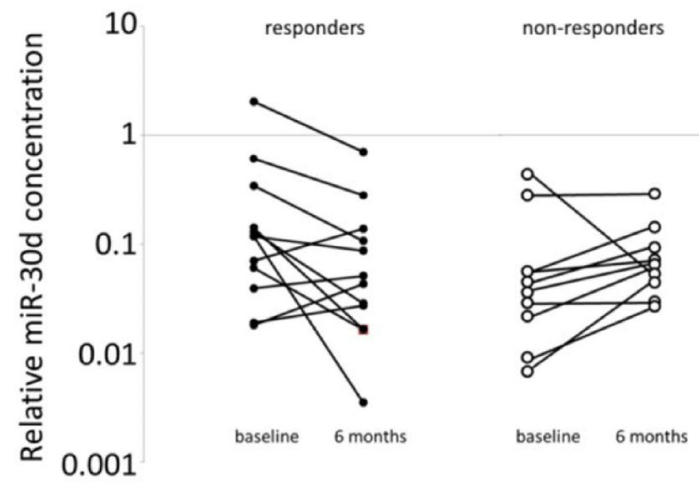

Figure 2.

Plasma miRNAs as biomarkers of response to CRT. A. Univariate logistic regression to determine miRNAs and clinical variables associated with favorable LV function post-CRT (increase in LVEF $210 \%$ ). B. Correlation between EF increase and miR-30d level. Associations is statistically significant with Spearman coefficient of $0.39, \mathrm{p}=0.01$ for miR-30d. Correlation between miR-30d and change in EF remained significant with exclusion of either outlier point ( $\mathrm{p}=0.01)$, but was borderline significant with exclusion of both extreme values (Spearman rho=0.31, $\mathrm{p}=0.058$ ). C. Probability of $10 \%$ improvement in 
EF plotted against log (miR-30d concentration) Logistic regression remained significant even after removal of extreme miR-30d values noted in $2 \mathrm{C}(\mathrm{p}=0.03)$. D. ROC curve for prediction of CRT response (as assessed by $10 \%$ improvement in EF) by miR-30d levels compared to prediction of response by QRS duration. $\mathbf{E}$ miR-30d levels significantly decrease by six months with CRT response but are unchanged in non-responders among 21 additional heart failure patients. 
A

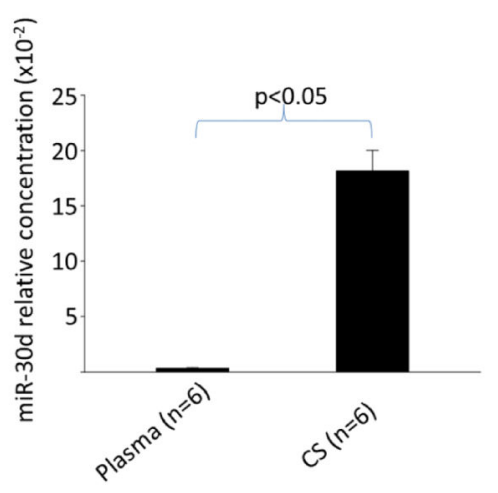

C

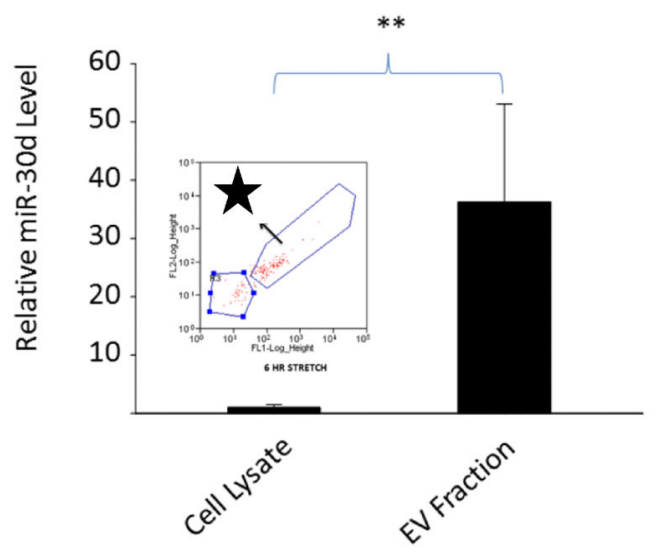

E

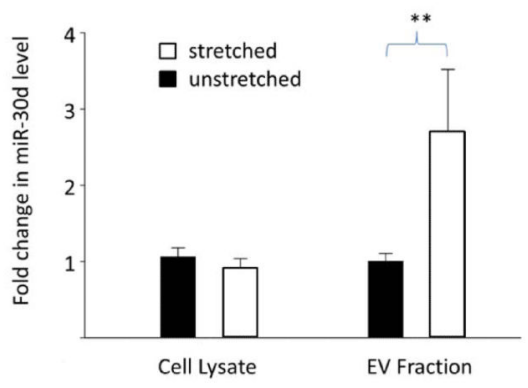

B

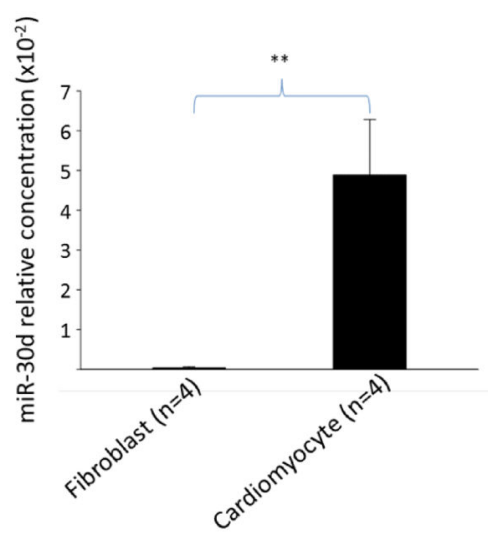

D

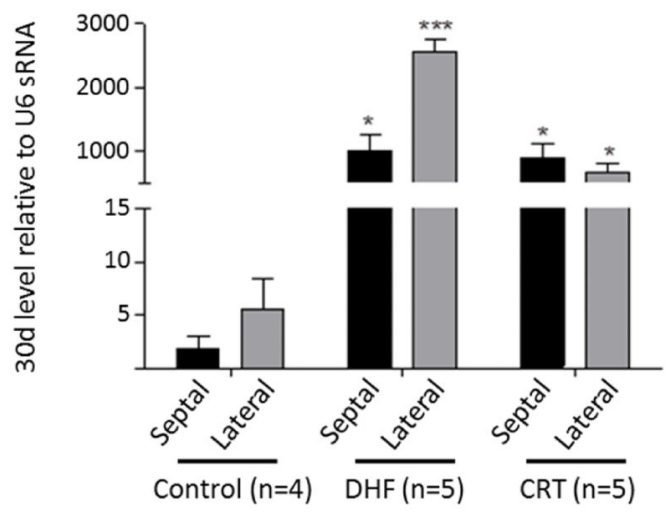

F

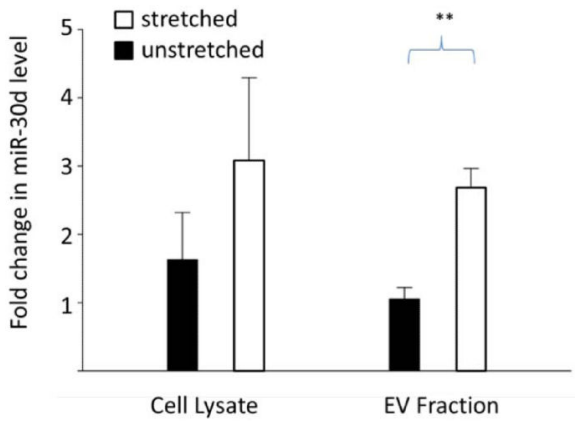

Figure 3.

Origin and regulation of miR-30d. A. miR-30d levels normalized to C-elegans spike-in miR-39) in samples from the coronary sinus and peripheral plasma in the same human subjects at the time of CRT implantation $(n=6)$. B. Cellular miR-30d level in cultured neonatal rat ventricular CMs versus cardiac fibroblasts (normalized to U6snRNA). $\mathbf{C}$. miR-30d is significantly enriched in the extracellular vesicle fraction (purified by ultracentrifugation followed by FACS sorting) relative to cellular lysates ( $n=3$ experiments). Inset FACS sorting of EV fraction following ultracentrifugation identifies a population of 
acridine orange positive particles with size $<200 \mathrm{~nm}$. D. miR-30d levels in the myocardium in a canine model of dyssynchronous heart failure $\left(\mathrm{HF}_{\mathrm{DYS}}\right)$ with subsequent resynchronization (CRT) compared to control. MiR-30d levels were measured in both the septal and lateral walls in control, $\mathrm{HF}_{\mathrm{DYS}}$ and CRT dogs. MiRNA levels were compared in each individual canine lateral versus septal wall by paired t-test, and between CRT, DHF and control animals by unpaired-t test. * denotes $\mathrm{p}<0.05$ relative to control septum; *** denotes $\mathrm{p}<0.05$ relative to DHF septum. miR-30d levels were normalized to U6snRNA (see Fig. S4). E. After 6 hours of cyclic stretch at $10 \mathrm{~Hz}$ on an elastic membrane, miR-30d levels are unchanged in $\mathrm{CM}$ cell lysate ( $\mathrm{n}=4$ experiments), but are significantly increased in the EV fraction. F. After 24 hours of stretch, levels of miR-30d remain elevated in the EV fraction. Levels of 30d are normalized to U6snRNA. For both EV fractions and cell lysates, fold change relative to unstretched fraction (EV or cell lysate) is shown. For all subfigures, ** denotes $\mathrm{p}<0.05$ by $\mathrm{T}$-test for normally distributed samples, and by Mann-Whitney for all others unless otherwise specified. 


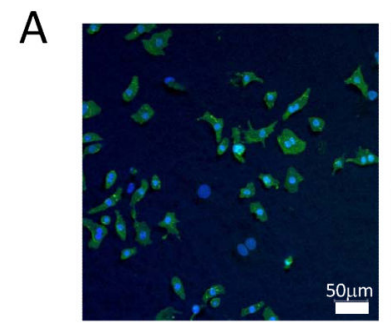

Scrambled miRNA

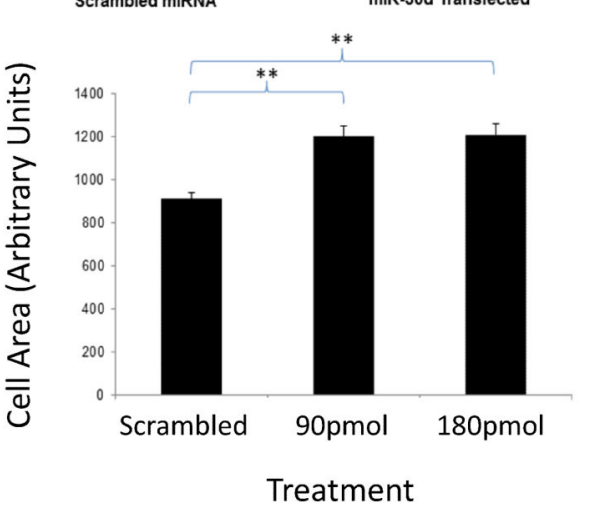

B

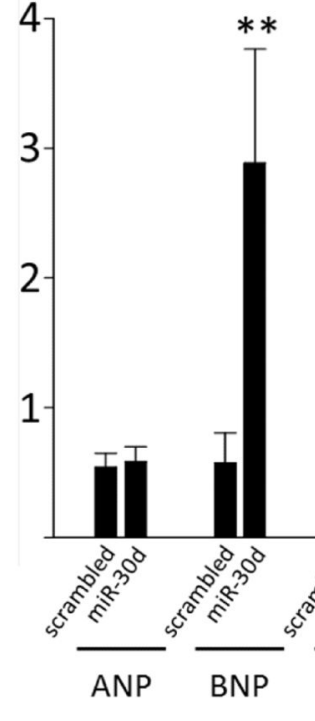

miR-30d Transfected

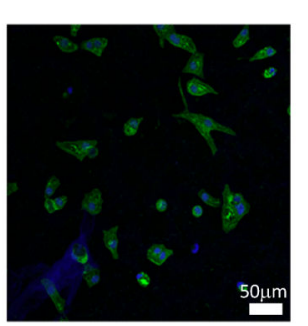

C
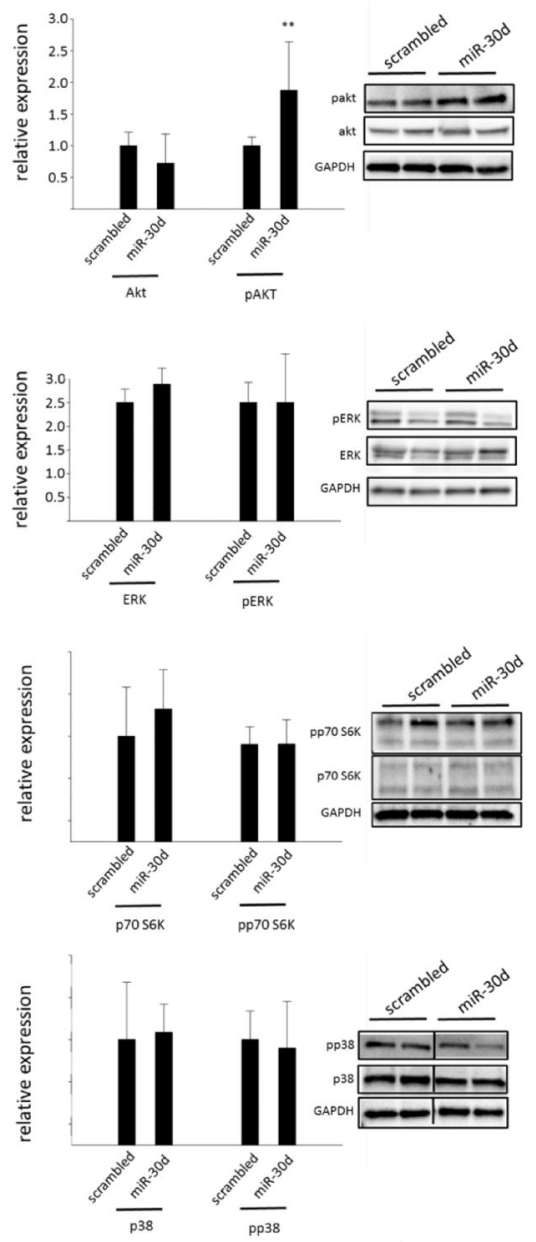

Figure 4.

miR-30d mediates adaptive hypertrophy in cultured CMs. A. Cultured rat CMs were transfected with either a sham or a miR-30d mimic using a liposomal delivery system, stained with a-actinin, and imaged with confocal microscopy. CM size was traced and quantitated using Image J software. Bar graph shows average of 150-290 cells in each group. *: p < 0.05. Scale bar: $50 \mu \mathrm{m}$. B. mRNA levels of gene markers of hypertrophy/ fibrosis as assessed by qRT-PCR. Results were normalized to house-keeping gene HPRT. **: $\mathrm{p}<0.05, \mathrm{n}=3$ experiments. C. miR-30d effects on hypertrophic signaling pathways: 
Protein lysates from CMs subjected to miR-30d mimic or scramble transfection for $24 \mathrm{hrs}$ were harvested and assessed for levels of phospho- and total levels of kinases implicated in cardiac hypertrophy as indicated. $\mathrm{n}=4$ independent experiments for each, $* *$ denotes $\mathrm{p}<0.05$ for comparison of pAkt in miR-30d and scramble transfected cells. 
A

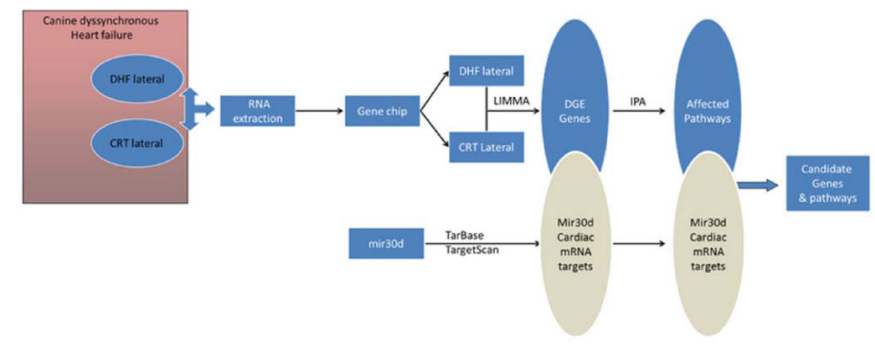

B

Dopamine Receptor Signaling PISK/AKT Signaling

Feedback in cAMP Signaling Cardiac ??-adrenergic Signalin RAR Activation

Protein Kinase A Signaling Aldosterone Signaling in Epithelial Cells CDK5 Signaling IIK Signaling

RhoA Signaling

Production of Nitric Oxide and ROS

Insulin Receptor Signaling

PTEN Signaling

ERK/MAPK Signalin

Netrin Signaling

Cardiovascular System

Breast Cancer Regulation by Stathmin

Integrin Signaling

CD27 Signaling in Lymphocytes

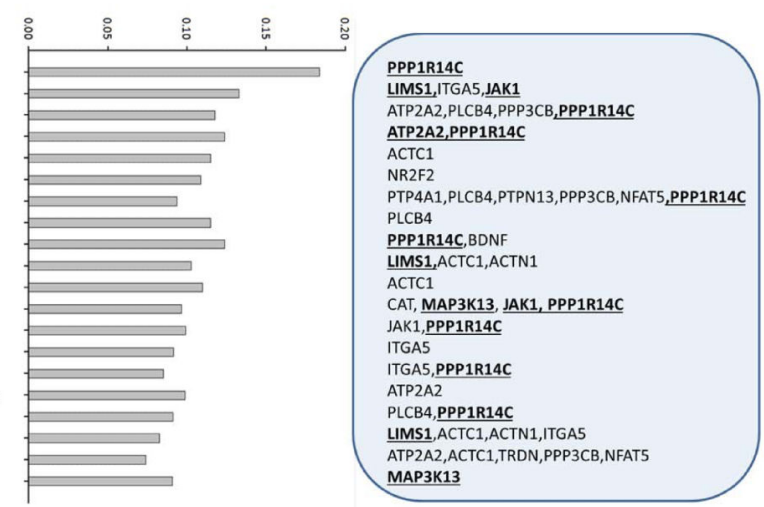

C

D
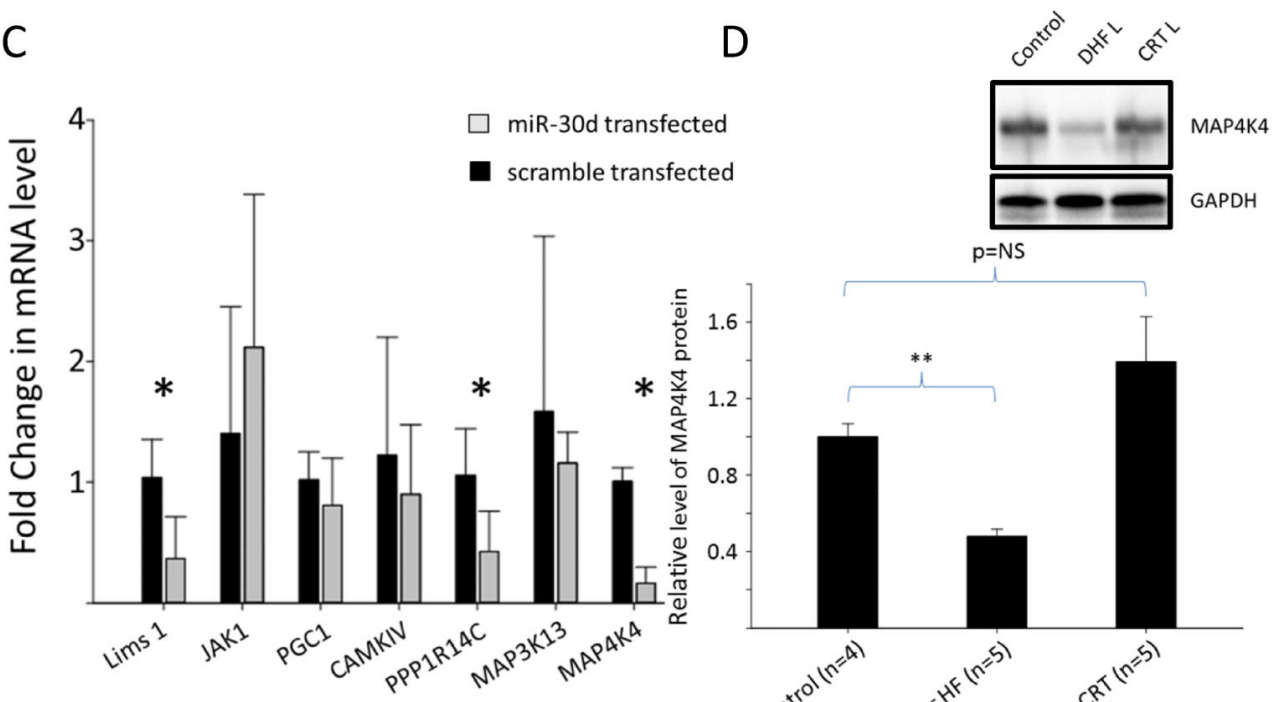

Figure 5.

LIMMA and IPA analysis identifies potential miR-30d gene targets. A. Schema of bioinformatics analysis to identify miR-30d targets in $\mathrm{HF}_{\mathrm{DYS}}$. Microarray gene expression data from the $\mathrm{HF}_{\mathrm{DYS}}$ and CRT lateral walls was subjected to LIMMA analysis to generate a set of differentially expressed (DGE Genes) genes. Within this set miR-30d putative targets were identified using Tarbase and Targetscan algorithms. IPA (Ingenuity Pathways Analysis) was then used to generate a set of predicted signaling pathways. B. List of pathways ranked by predicted magnitude of change. The grey box to the right identifies the 
putative miR-30d target gene. Bold and underlined molecules were selected for further investigation, based on the signaling pathways involved and/or their involvement in multiple pathways. C. miR-30d target genes of interest were evaluated in neonatal rat CMs that were transfected with scramble or miR-30d mimic. *: $\mathrm{p}<0.05$. D. Western blot analysis and quantitation of MAP4K4 protein levels in the dog dyssynchronous heart model (average of $\mathrm{n}=4$ dogs). Inset shows a representative Western blot. Levels were normalized to GAPDH. 
A

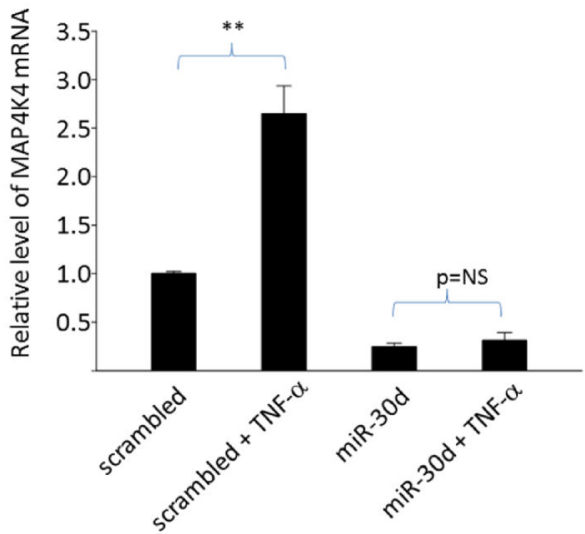

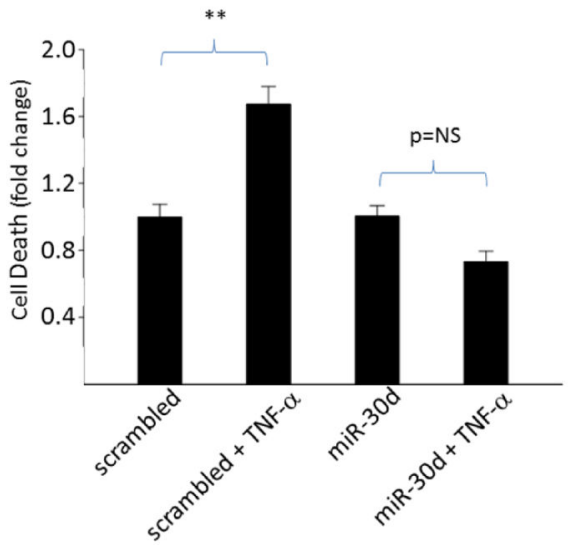

$B$

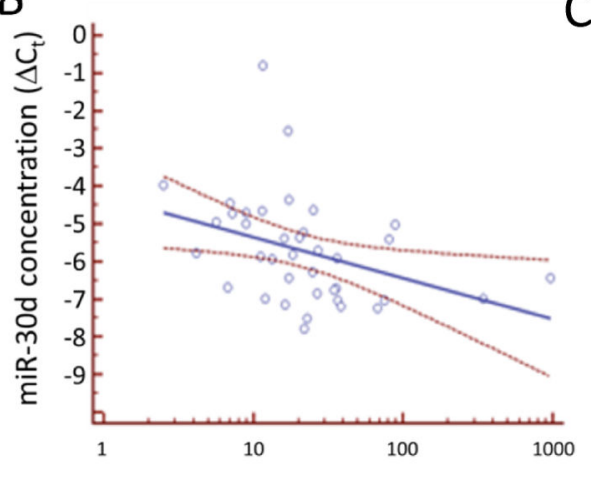

hs-TnT (concentration)
C

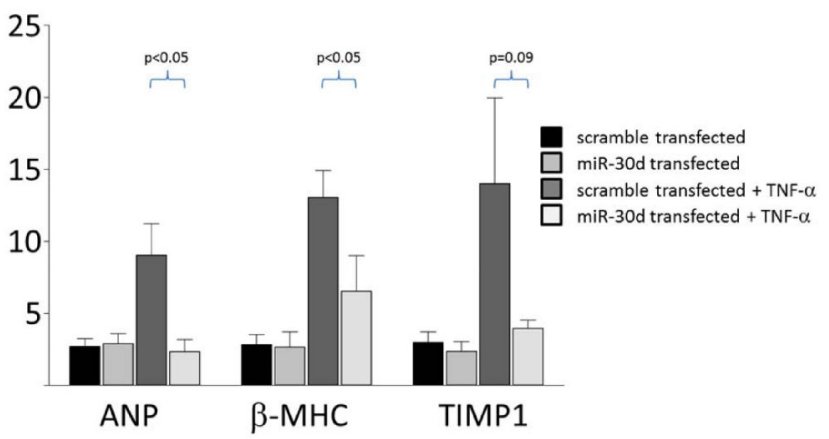

D

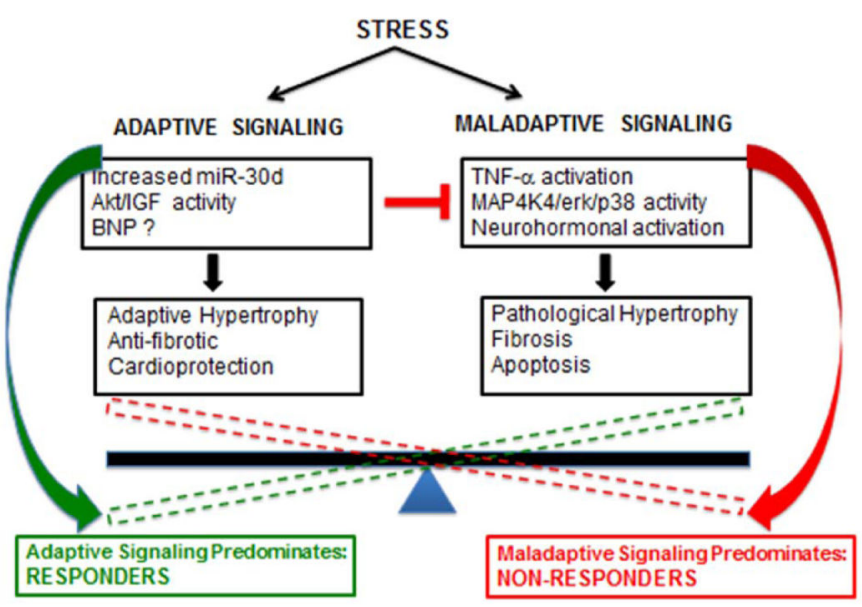

Figure 6.

miR-30d is cardio-protective against apoptosis by modulating signaling downstream of TNF-a. A. MAP4K4 mRNA levels in cultured rat CMs transfected with miR-30d mimic or scramble control and treated with TNF-a ( $25 \mathrm{ng} / \mathrm{mL}$ for 24 hours) ( $\mathrm{n}=5$ experiments), left panel; and level of apoptosis, as assessed by cell death assay ELISA kit in CMs transfected with miR-30d mimic or scrambled control and treated with TNF- $a$ as described above $(n=4$ experiments), right panel. $* *: \mathrm{p}<0.05$ for comparison of TNF-a treated and untreated scramble transfected cells. B. Levels of ANP, $\beta$-MHC and TIMP mRNA levels in cultured 
rat CMs transfected with miR-30d mimic or scrambled control treated as in (A). C miR-30d levels are correlated with baseline TnT levels, $\mathrm{r}=-0.51, \mathrm{p}=0.0001$ for the association. $\mathbf{D}$. Schematic for our proposed mechanism of miR-30d action in $\mathrm{HF}_{\mathrm{DYS}}$. 


\section{Table 1}

Baseline demographic, echocardiographic and biochemical characteristics of the study population, stratified by response to CRT. P values represent comparison between responder and non-responder population.

\begin{tabular}{|c|c|c|c|c|}
\hline Covariate & $\begin{array}{c}\text { All } \\
(N=40)\end{array}$ & $\begin{array}{c}\text { Non- } \\
\text { responder } \\
(\mathbf{N}=19)\end{array}$ & $\begin{array}{c}\text { Responder } \\
(\mathbf{N}=\mathbf{2 1})\end{array}$ & P value \\
\hline Age, years & $67.1 \pm 13.2$ & $68.7 \pm 12.9$ & $65.8 \pm 13.6$ & 0.50 \\
\hline Female gender, n (\%) & $8(20 \%)$ & $2(10 \%)$ & $6(29 \%)$ & 0.24 \\
\hline Ischemic HF etiology, n (\%) & $26(65 \%)$ & $15(79 \%)$ & $11(52 \%)$ & 0.10 \\
\hline Serum creatinine, $\mathrm{mg} / \mathrm{dl}$ & $1.44 \pm 0.48$ & $1.48 \pm 0.52$ & $1.40 \pm 0.47$ & 0.82 \\
\hline Prior revascularization, n (\%) & $20(50 \%)$ & $13(68 \%)$ & $7(33 \%)$ & 0.06 \\
\hline Baseline NYHA class & $3.0 \pm 0.3$ & $3.0 \pm 0.4$ & $3.0 \pm 0.3$ & 0.98 \\
\hline QRS duration on implant, msec & $174 \pm 27$ & $172 \pm 29$ & $175 \pm 26$ & 0.75 \\
\hline Bundle Branch Block, n (\%) & & & & 0.10 \\
\hline LBBB & $25(63 \%)$ & $9(47 \%)$ & $16(76 \%)$ & \\
\hline Paced & $15(37 \%)$ & $10(53 \%)$ & $5(24 \%)$ & \\
\hline Atrial fibrillation, $\mathrm{n}(\%)$ & $13(33 \%)$ & $6(32 \%)$ & $7(33 \%)$ & 1 \\
\hline Diabetes mellitus, n (\%) & $13(33 \%)$ & $6(32 \%)$ & $7(33 \%)$ & 1 \\
\hline Hypertension, n (\%) & $24(60 \%)$ & $13(68 \%)$ & $11(52 \%)$ & 0.35 \\
\hline \multicolumn{5}{|l|}{ Medication use, n (\%) } \\
\hline ACE inhibitor & $23(58 \%)$ & $13(68 \%)$ & $10(48 \%)$ & 0.22 \\
\hline ARB antagonist & $12(30 \%)$ & $4(21 \%)$ & $8(38 \%)$ & 0.31 \\
\hline Aldosterone antagonist & $14(36 \%)$ & $5(26 \%)$ & $9(43 \%)$ & 0.33 \\
\hline Beta-blocker & $37(93 \%)$ & $18(95 \%)$ & $19(90 \%)$ & 1 \\
\hline Digoxin & $8(20 \%)$ & $6(32 \%)$ & $2(10 \%)$ & 0.12 \\
\hline Diuretics & $32(80 \%)$ & $16(84 \%)$ & $16(76 \%)$ & 0.70 \\
\hline Aspirin use & $19(83 \%)$ & $14(74 \%)$ & $19(90 \%)$ & 0.82 \\
\hline Clopidogrel use & $9(23 \%)$ & $5(26 \%)$ & $4(19 \%)$ & 0.73 \\
\hline Coumadin & $18(45 \%)$ & $8(42 \%)$ & $10(48 \%)$ & 1.0 \\
\hline Transvenous implant, n (\%) & $38(95 \%)$ & $19(100 \%)$ & $19(90 \%)$ & 0.49 \\
\hline \multicolumn{5}{|l|}{ Baseline echocardiography } \\
\hline LV ejection fraction, $\%$ & $24.9 \pm 6.2$ & $25.9 \pm 6.4$ & $24.0 \pm 6.1$ & 0.36 \\
\hline $\begin{array}{l}\text { LV end-diastolic dimension, } \\
\mathrm{mm}\end{array}$ & $62.1 \pm 8.3$ & $61.6 \pm 8.0$ & $62.6 \pm 8.7$ & 0.72 \\
\hline $\begin{array}{l}\text { LV end-systolic dimension, } \\
\mathrm{mm}\end{array}$ & $53.4 \pm 8.5$ & $52.4 \pm 8.3$ & $54.7 \pm 8.8$ & 0.44 \\
\hline
\end{tabular}




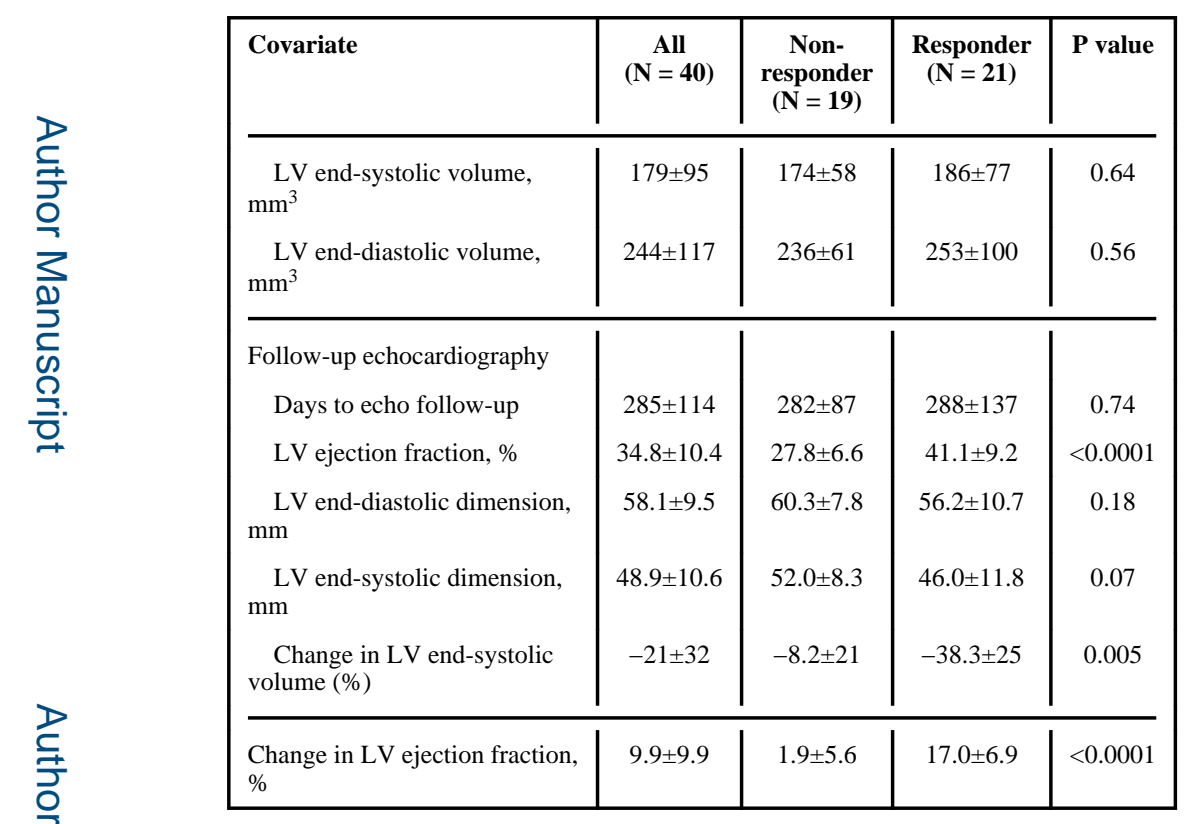

Abbreviations: HF = heart failure; NYHA = New York Heart Association; LBBB = left bundle branch block; RBBB = right bundle branch block; $\mathrm{ACE}=$ angiotensin converting enzyme; $\mathrm{ARB}=$ angiotensin II receptor blockade; $\mathrm{LV}=$ left ventricular. 


\section{Table 2}

Baseline demographic, echocardiographic and biochemical characteristics of the study population versus nonHF volunteers.

\begin{tabular}{|c|c|c|c|}
\hline Covariate $^{*}$ & $\begin{array}{c}\text { All } \\
(\mathrm{N}=\mathbf{4 0})\end{array}$ & $\begin{array}{c}\text { Non-HF } \\
\text { volunteers } \\
(\mathbf{N}=\mathbf{2 9})\end{array}$ & $P$ value \\
\hline Age, years & $67.1 \pm 13.2$ & $66.0 \pm 6.3$ & .62 \\
\hline Female gender, n (\%) & $8(20 \%)$ & $10(34 \%)$ & .27 \\
\hline Serum creatinine, $\mathrm{mg} / \mathrm{dl}$ & $1.44 \pm 0.48$ & $1.05 \pm 0.26$ & .0003 \\
\hline Prior revascularization, n (\%) & $20(50 \%)$ & $9(31 \%)$ & .14 \\
\hline Diabetes mellitus, n (\%) & $13(33 \%)$ & $1(3 \%)$ & .0005 \\
\hline Hypertension, n (\%) & $24(60 \%)$ & $15(52 \%)$ & .62 \\
\hline \multicolumn{4}{|l|}{ Medication use, n (\%) } \\
\hline ACE inhibitor & $23(58 \%)$ & $8(28 \%)$ & .02 \\
\hline ARB antagonist & $12(30 \%)$ & $5(17 \%)$ & .27 \\
\hline Beta-blocker & $37(93 \%)$ & $6(21 \%)$ & $<0.0001$ \\
\hline Diuretics & $32(80 \%)$ & $2(7 \%)$ & $<0.0001$ \\
\hline \multicolumn{4}{|l|}{ Baseline echocardiography } \\
\hline LV ejection fraction, $\%$ & $24.9 \pm 6.2$ & $68.6 \pm 5.6$ & $<0.0001$ \\
\hline $\begin{array}{l}\text { LV end-diastolic dimension, } \\
\mathrm{mm}\end{array}$ & $62.1 \pm 8.3$ & $43.4 \pm 4.5$ & $<0.0001$ \\
\hline $\begin{array}{l}\text { LV end-systolic dimension, } \\
\mathrm{mm}\end{array}$ & $53.4 \pm 8.5$ & $27.4 \pm 3.6$ & $<0.0001$ \\
\hline
\end{tabular}

Abbreviations: $\mathrm{HF}=$ heart failure; NYHA = New York Heart Association; LBBB = left bundle branch block; RBBB = right bundle branch block; $\mathrm{ACE}=$ angiotensin converting enzyme; $\mathrm{ARB}=$ angiotensin II receptor blockade; LV = left ventricular.

Values reported where available $(\mathrm{N}$ ^29). LVEF $(\mathrm{N}=18)$ and LV dimensions $(\mathrm{N}=17)$ reported where available. 
Table 3

Univariable logistic regression for prediction of CRT response (defined as increase in LV ejection fraction $\geq$ $10 \%$ ). Odds ratios represent odds of response to CRT.

\begin{tabular}{|l|c|c|c|}
\hline \multirow{2}{*}{ Covariate } & \multicolumn{3}{|c|}{ CRT Response } \\
\cline { 2 - 4 } & OR & 95\% CI & P value \\
\hline Age & 0.98 & $0.94-1.03$ & 0.49 \\
\hline Female gender & 3.40 & $0.59-19.46$ & 0.17 \\
\hline Ischemic HF & 0.29 & $0.07-1.19$ & 0.09 \\
\hline Creatinine & 0.70 & $0.19-2.57$ & 0.59 \\
\hline Prior revascularization & 0.23 & $0.06-0.87$ & 0.03 \\
\hline Pre-implant QRS & 1.00 & $0.98-1.03$ & 0.74 \\
\hline AF & 1.08 & $0.29-4.08$ & 0.91 \\
\hline Diabetes mellitus & 1.08 & $0.29-4.08$ & 0.91 \\
\hline Beta-blocker use & 0.52 & $0.04-6.34$ & 0.61 \\
\hline Digoxin use & 0.23 & $0.04-1.31$ & 0.10 \\
\hline Diuretic use & 0.60 & $0.12-2.94$ & 0.53 \\
\hline Hypertension & 0.51 & $0.14-1.85$ & 0.30 \\
\hline ACE-I use & 0.42 & $0.12-1.53$ & 0.19 \\
\hline ARB use & 2.31 & $0.56-9.47$ & 0.25 \\
\hline Aldosterone antagonist use & 2.10 & $0.55-8.00$ & 0.28 \\
\hline Baseline LVEF & 0.95 & $0.86-1.06$ & 0.35 \\
\hline Baseline LVEDD & 1.02 & $0.94-1.10$ & 0.72 \\
\hline Baseline LVESD & 1.03 & $0.95-1.12$ & 0.43 \\
\hline miR-30d & 2.32 & $1.16-4.67$ & 0.02 \\
\hline miR-29c & 1.23 & $0.82-1.84$ & 0.32 \\
\hline miR-142-5p & 1.98 & $1.17-3.37$ & 0.01 \\
\hline miR-766 & 1.85 & $1.09-3.16$ & 0.02 \\
\hline Abeve & \\
\hline
\end{tabular}

Abbreviations: $\mathrm{OR}=$ odds ratio; $\mathrm{CI}=$ confidence interval; $\mathrm{HF}$ = heart failure; $\mathrm{AF}=$ atrial fibrillation; $\mathrm{ACE}-\mathrm{I}=$ angiotensin converting enzyme; $\mathrm{ARB}=$ angiotensin II receptor blocker; $\mathrm{LVEF}=\mathrm{LV}$ ejection fraction; $\mathrm{LVEDD}=\mathrm{LV}$ end-diastolic dimension; $\mathrm{LVESD}=\mathrm{LV}$ end-systolic dimension. miRNA levels are log-transformed. NS = non-significant. 